\title{
Quality Assessment of Cereals using the Hilbert Transform
}

\author{
S Ananthi ${ }^{1 *}$, A Arun ${ }^{1}, \mathrm{~K}$ Sudhamathy ${ }^{1}, \mathrm{KR}$ Balaji $^{1}$ \\ ${ }^{1}$ Department of Network Systems and Information Technology, University of Madras, Guindy campus, Chennai, India
}

Received: August 02, 2016; Accepted: August 16, 2016; Published: September 05, 2016

*Corresponding author: S Ananthi, Associate Professor \& Head-in-Charge, Department of Network Systems \& Information Technology, Guindy Campus, University of Madras, Chennai -600 025, India, Tel: +044 22202767; Fax: +044-22352494; E-mail: ananthipradeep84@gmail.com; ananthibabu@unom.ac.in

\begin{abstract}
In work relating to optics, dispersion relations have provided an insight into the inter relationship between the two parts of the signal passing through an optical medium-the absorptive and the dielectric parts. This relation is given by the analytic function satisfying the Cauchy-Riemann conditions. Measurements of dielectric constant and dielectric loss of an intrinsic material indicate that the two are similarly related and the total function $\sigma+\varepsilon$ are an analytic function of frequency or time. Using this property, whenever there is any nonlinearity due interference from impurities or other effects, the same can be eliminated. Measurements for materials such as food powders, cellulose, cereals and the like can utilize this property to estimate the quality of the materials. The determination of the quality of a grain, say, rice or wheat, is generally considered as a difficult measurement parameter. However, in the proposed method, this quality has been traced to the value of the capacitance in a cell packed with the grains. Such small capacitance values are not easily measurable in presence of a large parallel conductance, which is due to absorbed and inherent water content. Further, there is also a non-linearity in the ionic conductance part. Since the capacitance alone has to be isolated for assessing the quality, the problem is that of a measurement of a small value of capacitance which is in parallel with a high nonlinear ionic conductance. This paper describes the use of a suitable transformer ratio arm bridge. The data processing is done by digital signal processing. The non-linearity problem was solved by using digital quadrature detection of the bridge signal along with the use of Hilbert Transform. The method is explained and results are shown to be repetitive and decisive in assessing the quality of cereals.
\end{abstract}

Keywords: Analytic Signal; Hilbert Transform; Non Linear Signal Processing; Capacitive Cum Conductive Transducers; Quality Assessment of Cereals; Transformer Ratio Arm Bridge

\section{Introduction}

In transducers of the capacitive cum conductive type, invariably non linear conductance contributes to an erroneous measurement of the Capacitance part. For instance, while the measurement of moisturized transformer oil's dielectric properties are measured with a transformer ratio arm bridge, the separation of the capacitive component from the non-linear ionic conductance appears to mask the results considerably. So, a study was made of the composite signal component due to the complex impedance $\mathrm{R}+\mathrm{jX}$ as an analytic function.
Due to the effects dependent on impurities and stray causes, the two components of this impedance function, the conductance and the capacitance, do not fit into an exact analytic function. Mainly, the effects of non-linearity in conductance exhibited by the impure parts are to be eliminated. Since the capacitance part is mostly affected only by additive stray effects which can be taken into account (by a dummy measurement with no material between electrodes), the conductance part cannot be easily removed of the impure, interfering components.

In the case of ionic conductivity, such as the moisture with salt absorbed in the oil specimen, the same is having a non-linear ohmic relation and the effect is to introduce a wrong component into the separated signal for the capacitance component from the bridge cell.

So, in order to resolve this problem, the fact that the Hilbert transform is the link between the two components of a linear analytic signal [1], has been used and found to give the corrective procedure. Because the signal from the capacitive component has no non-linearity (except under high electric fields, for the case of the transformer oil bridge measurement), the Hilbert transform of the bridge signal for an excitation burst to the bridge provides the linear part of the conductance component, such as

$$
R_{\text {lin }}+j X
$$

And by simple arithmetic, the non linearity due to the conductancecan be eliminated.Transducersbased onconductance or capacitance measurements for physical quantities are quite many and well known. Many physical phenomena involve these two properties. In optical measurements, substances absorb light and refraction also takes place. The net property is a complex refractive index, a complex function of frequency, given by

$$
v(\omega)=\sigma+j \varepsilon
$$

Where $\sigma$ denotes the conductance and $+j$ the dielectric constant. If such a complex function is analytic, it satisfies the Kramers-Kronig relations [2] given by

$$
X_{1}(\omega)=\frac{1}{\pi} P \int_{-\infty}^{\infty} d \omega^{1} \frac{X_{2}\left(\omega^{1}\right)}{\omega^{1}-\omega}
$$


And

$$
X_{2}(\omega)=-\frac{1}{\pi} P \int_{-\infty}^{\infty} d \omega^{1} \frac{X_{2}\left(\omega^{1}\right)}{\omega^{1}-\omega}
$$

The above relation applies for any function $\chi(\omega)=\chi_{1}(\omega)+$ $j \chi_{2}(\omega)$ analytic in the upper half plane which vanishes as $|\omega| \rightarrow \infty$. $P$ denotes the Cauchy principal value of the integral.

The meaning of this is that the real and imaginary parts of the analytic function, for example, the absorbance and the dielectric constant, are not independent. Further, they are related by the integral which is the definition of the Hilbert Transform [3]. In reality, the two signal components on the right hand side of equation (1) are actually obtained from one causal phenomenon.

\section{Methods}

For causal signals, the two components of the signal are always related by the Hilbert transform. Thus, the real part signal is the Hilbert transform of the imaginary part and vice versa. Hilbert transform is a filter that converts sines into cosines and is called a " $90^{\circ}$ phase-shift filter" or a "quadrature filter". The definition of the Hilbert transform is easily understood in the frequency domain than in time. The Hilbert transform is just obtained by a filter $Q(\omega)$ which gives a phase shift of $-\pi / 2$ for all the frequencies which are positive and by $+\pi / 2$ for all that are negative (Figure 1).

$$
Q(\omega)=\frac{-j \omega 1}{|\omega|}=-j \operatorname{sgn} \omega
$$

The time domain discrete form is obtained through the inverse Fourier Transform and is

$$
q_{n}=\left\{\begin{array}{l}
\text { ofor } n \text { even } \\
\frac{-2}{\pi n} \text { for } n \text { odd }
\end{array}\right.
$$

\section{Phase sensitive detection}

In transducers of the capacitive cum conductive type, invariably non linear conductance contributes to an erroneous measurement of the capacitance part. As an example, in the measurement of transformer oil's dielectric properties using a transformer ratio arm bridge, we get the two components, namely the capacitance and the conductance. These components are separated from the signal output voltage from the bridge using phase sensitive detection. Here, two detecting circuits are involved; one gives the in-phase component, the other gives the imaginary or Quadrature component, as $\chi(\omega)=\chi_{1}(\omega)+j \chi_{2}(\omega)$. Here the $\chi$ denotes the impedance of the measuring cell.

If the signals are phase sensitively detected, there arise the two components -- in-phase or the real part and the out of phase or quadrature part.

If $\mathrm{V}_{\mathrm{d}}$ is the detected output signal voltage, then,

Direct or in-phase component

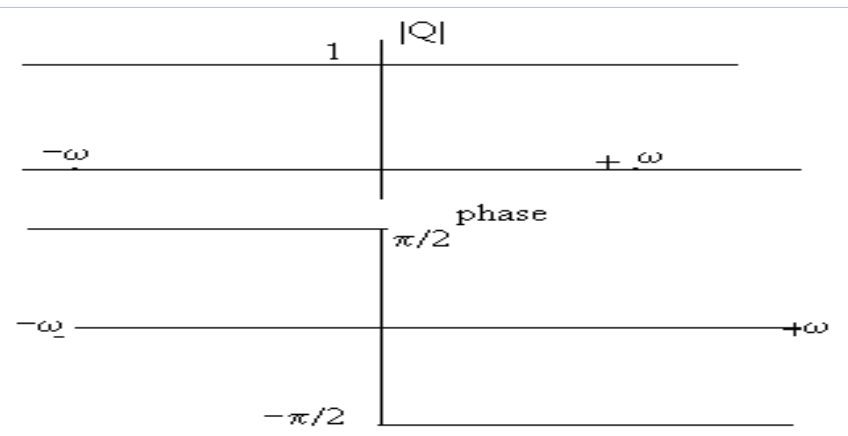

Figure 1: The Hilbert Transform transfer function. Magnitude is unchanged, phase is \pm 90 o.

$$
V_{\text {real }}=\int_{0}^{2 \pi} V_{d} \operatorname{Sin} \omega t d t
$$

Quadrature Component

$$
V_{\text {Quad }}=\int_{0}^{2 \pi} V_{d} \operatorname{Cos} \omega t d t
$$

When the properties are measured for a pure substance, the two components will give the conductance and capacitance respectively. However, this separation of the capacitive component from the conductance is subject to errors whenever there are interactive disturbances, causing one component to interfere with the other. One reason is because of the nonlinear nature of ionic conductance and another is dead time. Nonlinearity in the conductance component can arise when the current to voltage relationship is not linear due to the property of ionic conduction [4]. So, when we measure a capacitance change it appears on the conductance and vice versa, with the result that one appears to mask the other. So, we cannot infer that the quadrature component is only due to capacitance.

At a particular frequency, the capacitance $C$ gives rise to a reactance $X$ at a frequency $\omega$ given by $X=1 / \omega C$, while the conductivity gives rise to a resistance $R$. So, a study was made of the composite signal component due to the complex impedance $R+j X$ as an analytic function. Due to the effects dependent on impurities and stray causes, the two components of this impedance function, the conductance and the capacitance, may not fit into an exact analytic function. Mainly, the effects of nonlinearity in conductance exhibited by the impure parts are to be eliminated. The capacitance part is not so much affected as does the conductance, except by additive stray effects which are correctible (by shielding and using guard electrodes and making dummy measurement with no material between electrodes). But the absorption (or conductance) component of a substance cannot be easily separated from the impure, interfering components.

In the case of ionic conductivity, such as the moisture with salt absorbed in the oil specimen, the same is having a nonlinear ohmic relation (Figure2) and its effect is to introduce a wrong component into the separated signal for the capacitance 
component from the bridge cell.

So, in order to resolve this problem, the fact that the Hilbert transform is the link between the two components of a linear analytic signal, has been used and found to give a fairly corrective procedure. The capacitive component has no non-linearity (except under very high electric fields, for the case of the transformer oil bridge measurement). The resistive component can be linked to it theoretically, by its Hilbert transform. If this is represented as $\mathrm{R}_{\operatorname{lin}}$, then, under ideal conditions, the bridge signal provides the linear part of the conductance component together with the capacitive component, as

$$
R_{\text {lin }}+j X
$$

But the actual value of $R$ obtained by the measurement will be different from this theoretical $R_{\text {lin }}$ value. The difference is the disturbing component due to nonlinearity and impurity.

\section{Experimental set-up using transformer ratio arm bridge and measurement cell}

In the set up of measurements based on this principle, alternating current bridges are employed. Figure 2 shows the Blumelein or transformer ratio arm bridge used because it is particularly suitable in this case since this set up is easily shielded from stray effects of capacitance. Here, $Y_{1}$ and $Y_{2}$ are the admittances of the two arms of the bridge. The transformer has a centre tapped secondary and we get two voltages $E_{1}$ and $E_{2}$ from it. A sinusoidal excitation is applied to the transformer with a frequency of $5 \mathrm{KHz}$ with a provision to vary it over a range of 1 to $10 \mathrm{KHz} . \mathrm{I}_{1}$ and $\mathrm{I}_{2 \text { are }}$ the currents from the top and bottom of the secondary winding. The difference current $\left(\mathrm{I}_{1}-\mathrm{I}_{2}\right.$ flows through the shunt resistor $\mathrm{R}_{\mathrm{s}}$ in the line connecting to the centre tap. When the bridge arms comprising of the standard capacitor and resistor and the transducer cell are balanced, any change in the transducer parameters due to the physical quantity of interest varying would produce the unbalance current. This unbalance is obtained across the shunt resistor $R_{s}$ as the signal. Two phase sensitive detectors are used to separate the in-phase and quadrature components of this voltage drop. For this, the two reference voltages to the phase sensitive detectors are the Sine and Cosine signals of the waveform of transformer voltage,

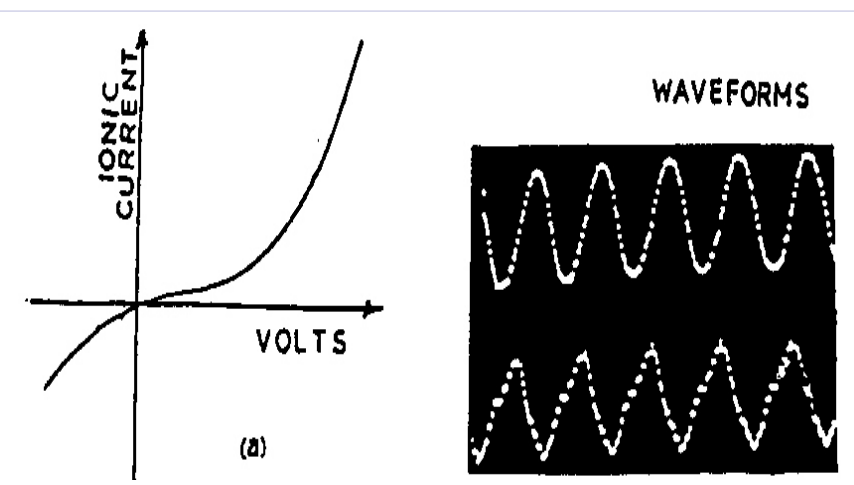

Figure 2: a): The ionic conductivity Volt-ampere relation and b): its effect on waveform of detector output. Top wave: bridge applied volts, bottom: detector output. the latter is got by the integration using an analog integrator as shown in figure 3 .

For a linear transducer, the real and quadrature phase sensitive detector signals are as shown by the phasor diagram insert in the figure 2. One gives the changes of conductance and the other of the capacitance. If $\mathrm{R}_{\mathrm{s}}$ is the shunt resistor, $A$ the amplifier gain at the frequency $\omega, \Delta G$ and $\Delta B$ are the conductance $G$ and capacitive susceptance $B$ detected, and $\mathrm{E}_{1}$ is the voltage of the transformer secondary per phase, then we get the output voltages shown as

Voltage of the phase sensitive detector -1 (in phase) $=\mathrm{A}$ $\mathrm{R}_{\mathrm{s}} \mathrm{E}_{1} \Delta \mathrm{G}$

Voltage of the phase sensitive detector -2 (Quadrature) $=\mathrm{A}$ $\mathrm{R}_{\mathrm{s}} \mathrm{E}_{1} \omega \Delta \mathrm{C}=\mathrm{AR}_{\mathrm{s}} E_{1} \Delta B$

However, when there is nonlinearity in the conductance the two outputs mix together and we get a capacitance change wrongly due to a conductance change from the two detector output.

\section{Digital phase sensitive detection}

In order to use the technique based on the analytic signal integration, it is necessary to employ digital signal processing and hence it is convenient to perform the phase sensitive detection [5] also digitally based on samples of bridge output signal measured with a fast Analog Digital Converter (ADC). A schematic is given in figure 4.

Here, again, the signal is picked from the detector shunt resistor and converted to digital signal. Also, the reference signal, taken from the transformer secondary is digitized and this is reference used for digital phase sensitive detection. was applied, resulting in a detector arm signal. Actually there are two channels for the ADC, one for the signal across the shunt as shown and another for the reference (not shown). Both the excitation reference and the output from the bridge were digitized for this purpose at a sampling rate of at least 60 times more than that of the bridge input frequency $(1-10 \mathrm{KHz})$. When detection is done by numerical integration of the digitized output signal and the carrier signal, we need many samples per one period of the carrier wave. The accuracy of the final value of the $\Delta \mathrm{G}$ and $\Delta \mathrm{B}$ depends on this number.

These digitized signals are analyzed with in-phase and quadrature detectors, performed by integrating the sample values over a fixed period of time, which can be in terms of complete sine wave samples, say 100. If the sampling time is $t_{s}$ the number $N$ obtained by dividing 100 times $T_{s}$ (where $\mathrm{T}_{\mathrm{s}}$ is the carrier frequency period $\left(=1 / f_{s}\right)$ ) by $t_{s}$, should be an even integer. If $V_{1}$ refers to the digitized carrier voltage and $V_{2}$ digitized bridge output signal, then the in-phase and quadrature components are digitally obtained by the summation of the products

$$
V_{\text {real }}=\sum V_{1}[n] V_{2}[n]
$$

$V_{\text {quad }}=\Sigma V_{1}[n+K] V_{2}[n]\left(\ldots K\right.$ is the samples in $90^{\circ}$ of the carrier wave) ...(9) 


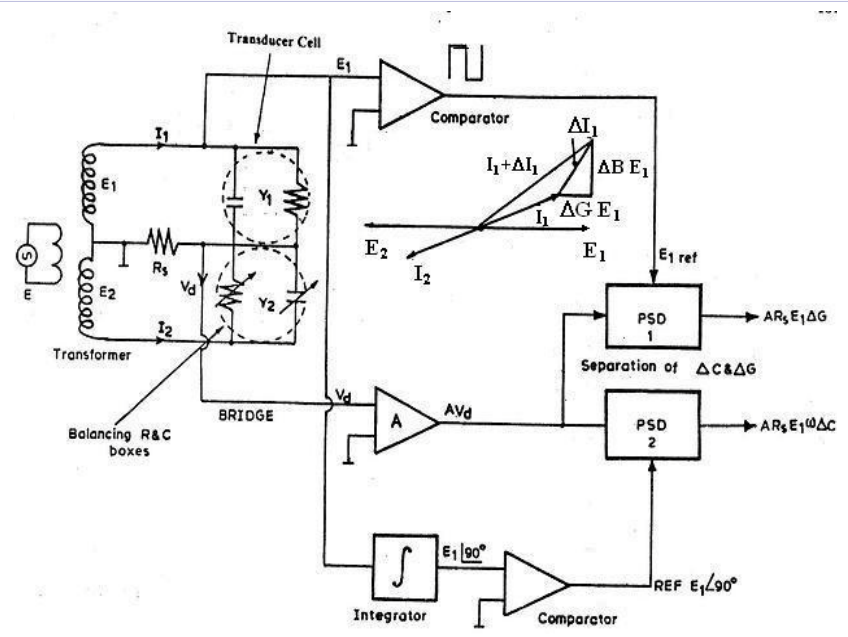

Figure 3: Shows the Transformer ratio arm (TRA) bridge set-up.

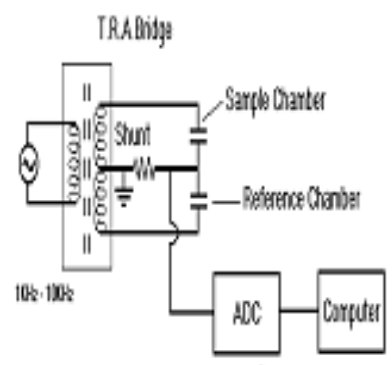

a)

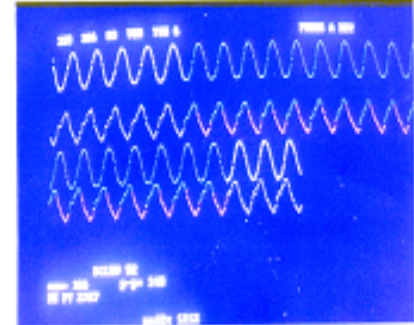

b)
Figure 4: 4a: The Method of measurement using Digital quadrature detection and $4 \mathrm{~b}$ : Showing the reference and detected signal waveforms from the TRA Bridge with the transducer.

Where the summation is for $N$ samples covering 100 full carrier frequency cycles.

The imaginary or quadrature component is obtained similarly.

The detected output has phase shift and distortion. The integration is by the sum of products mentioned in the equations 8,9 above

It is necessary to perform integration of the signals with the reference voltages now obtained by digital signal processing. The signals and the waveform of E1 are shown for many waves in figure 5 . The integration is therefore performed not just on one cycle but over a large number of cycles, in order to make it free from errors.

From the components of the signal so obtained, Hilbert transform is done digitally for both the in-phase and quadrature detected output voltages. These are used in evaluating the actual changes in capacitance and conductance. The advantage of using the Hilbert transform is to eliminate the stray effects and noise in the conductance as well as non-linearity which introduces harmonic components in the signals.

\section{Hilbert transform detection}

While equations (4) and (5) represent the phase sensitive detection normally performed, let us describe the use of the Hilbert transform for doing phase sensitive detection.

The quadrature component of the signal is shown in eqn. (10) below to be integral (over a carrier frequency period) of the carrier signal with the Transform of the detected output voltage $\mathrm{V}_{\mathrm{d}}$.

$$
\mathrm{V}_{\mathrm{H.Q}}=\int H\left\{V_{d}\right\} \operatorname{Sin} \omega t d t
$$

\section{( $H$ denotes Hilbert Transform)}

The samples of signals, the carrier frequency voltage, the detected bridge output and its Hilbert transform, for use in digital phase detection will look like (Figure 5).

The Hilbert transform phase detector extracts the phase error by using trigonometric computations. The Digital Carrier Oscillator used in this kind of phase detector produces two signals and in-phase signal $I=\cos \left(\omega_{0} t\right)$ and a quadrature signal $Q=\sin \left(\omega_{0} t\right)$.

If the input signal is a digital signal of the form

$$
u_{1}(t)=\cos \left(\omega_{0} t+\theta_{e}\right)
$$

Then by trigonometric operations,

$$
\begin{aligned}
& \operatorname{Cos} \theta_{e}=I u_{1}+Q u_{1}{ }^{\prime} \\
& \operatorname{Sin} \theta_{e}=I u_{1}{ }^{\prime}-Q u_{1}
\end{aligned}
$$

\section{Where ' $u$ ' represents the Hilbert transform of the signal $u$}

Since the analytic counterpart of the signal has to be determined for a discrete signal, the use of a Discrete Hilbert transform by Digital signal processing [4] done using Cizek's algorithm [5]. This evaluates the discrete Hilbert Transform of a sequence $\mathrm{x}(\mathrm{v})$ as the first summation being from $v=0$ to $\mathrm{N}-1$ and the second from $k=1$ to $(N-1) / 2$ if $\mathrm{N}$ is odd and (N/2 -1) if $\mathrm{N}$ is even, where $N$ is the number of samples. This can also be done through a matrix format for easy program in

$$
Y n_{=}-\{2 / N\} \sum x_{v} \sum \operatorname{Sin}(n-v) k(2 / N)
$$

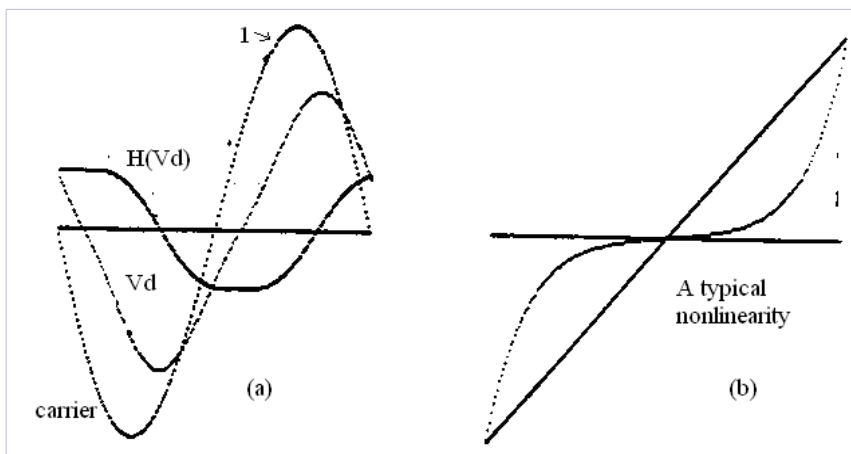

Figure 5: Vd is detector signal, Ref is reference voltage (E1), H(Vd) is the Hilbert transform of detector signal. The typical (assumed) nonlinear relation for which these waveforms apply is also shown. 


\section{Results}

The results of the measurements, when repeated with the bridge gave repeatable results among several sets of prepared samples from substances (Figure 6). The capacitance changes with addition of moisture content to the substance were also indicative of moisture content correctly.

The experiments used a Transformer Ratio Arm (TRA) bridge with a digitization bridge output and signal processing In identifying the substances among their groups, such as raw rice and boiled rice for example, the curve of capacitance change provided a clear indicator. With the Hilbert transform based detection, the curves were same with all sample preparations. Out of these, two results of practical importance are given below.

1. In rice varieties, the experiments were done in flat large packed cells with measured addition of pure water (Figure 7).

2. The measurements were able to reveal, by their corrected components of conductance/capacitances, how the expensive "Ponni" variety had better quality in comparison to fast yield plantation varieties of rice like IR-20, "Kuruvai $R$ " etc. The figure 9 shows the range of measured output with moisture
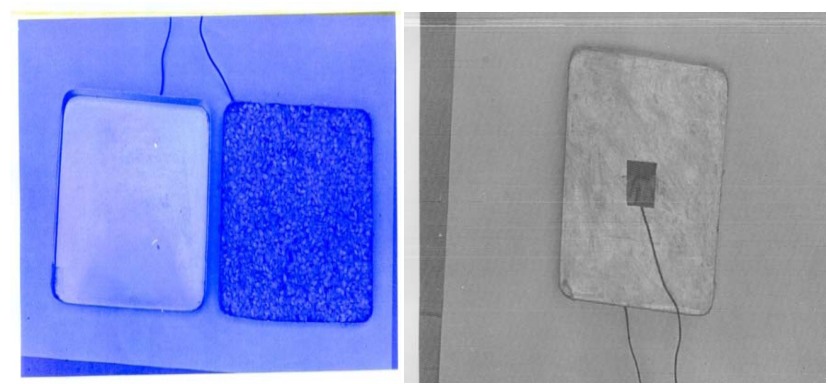

Figure 6: (Left) Showing the filling of grains in box capacitor and (right) closing it for setting up the measurement.

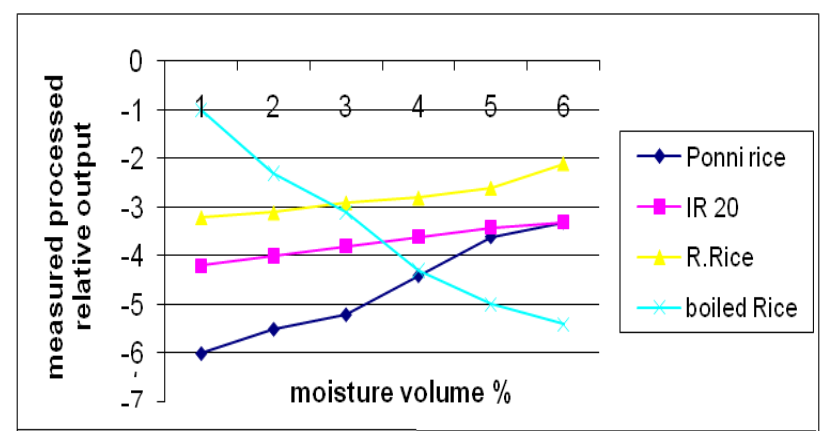

Figure 7: Showing the measurement of varieties of Rice in a packed moisturized cell using the T.R. A bridge with Hilbert transform based analytic for the varieties. The $\mathrm{Y}$ axis shows the change in percent value of $\Delta \mathrm{X}_{\mathrm{c}} / \mathrm{X}_{\mathrm{c}}$. It is to be noted that the higher priced rice has a larger range of variation. These high quality varieties are harvested with plantation duration of six months ("Ponni") while the "Kuruvai" type is a short duration (about 3 months) yielding variety. So, this range of variation in the curve is an indicator of the quality, the large range of variation occurring for the high priced rice variety. The Boiled rice is a type which is prepared by boiling the rice with husk and drying, with subsequent husk removal. Since the boiling causes the range of measured capacitance component to be more, the curve shows it accordingly, after the non-linearity correction using the equation 911]. The curve shows a different trend for this substance. We do not want to guess the physical reason for this. A calibration curve was prepared using substance cells with varying amounts of water added by weight and homogenized. The results were tabulated and programmed into the microcomputer. The system is for developing a direct reading instrument for quality assessment of grain samples.

\section{Conclusion}

The results proved to be linearly related because of the relationship of the causal signal of conductance and capacitance in a specimen with the help of the Hilbert Transform based Quadrature phase sensitive detection, performed by digital signal processing. The method is likely to be applicable for many other physical measurements using the principle of conductance or capacitance changes even in presence of nonlinearity precisely. Thus, we have developed a measurement scheme for tackling nonlinearity in transducers for quite many physical measurements, for which two examples have been provided.

\section{References}

1. Gold B, Opperheim AV, Rader CM. Theory and Implementation of the Discrete Hilbert Transform". Proc. Sym. Comp. Proc. in Communication. 1969;235-250.

2. Jackson JD. Classical Electrodynamics. 2nd edition. New York: Wiley. 1975;p:7.10

3. Padmanabhan K, Ananthi S. Hadamard transform based small capacitance transducer measurements. The Institution of Engineers (India), Div. Part of Jl. of Electronics and Telecommunication. 81:9-15.

4. DanieL Traficante D. Phase-Sensitive Detection. Part II: Quadrature Phase Detection. Concepts in Magnetic Resonance. 1990;2(4):151167.

5. Gold B, Opperheim AV, Rader CM. Theory and Implementation of the Discrete Hilbert Transform. Proc. Sym. Comp. Proc. in Communication. 1969;235-250.

6. Cizek V. Discrete Hilbert Transform. IEEE. Trans. on audio and Electroacoustics. 1970;18(4):340-44

7. Padmanabhan K, Ananthi S, Patel K. Portable Ozone Generator. EFY Magazine India. 32(6):46-52. 